\title{
Hybrid PET/CT and PET/MRI imaging of vulnerable coronary plaque and myocardial scar tissue in acute myocardial infarction
}

Authors:

- Stephanie Marchesseau, MSc, PhD, Clinical Imaging Research Centre, A*STAR-NUS Singapore

- Aruni Seneviratna, MBBS, Department of Cardiology, National University Heart Centre, Singapore

- A. Therese Sjoholm ${ }^{1}$, MSc, Clinical Imaging Research Centre, A*STAR-NUS, Singapore

- Daphne Liang Qin, Department of Cardiology, National University Heart Centre, Singapore

- Jamie X.M. Ho, Clinical Imaging Research Centre, A*STAR-NUS, Singapore

- Derek J. Hausenloy, MBChB, PhD, FRCP, FACC, FESC, (1) The Hatter Cardiovascular Institute, University College London, London, UK (2) The National Institute of Health Research University College London Hospitals Biomedical Research Centre, London, UK (3) National Heart Research Institute Singapore, National Heart Centre Singapore, Singapore. (4) Cardiovascular and Metabolic Disorders Program, Duke-National University of Singapore Medical School, Singapore

- David W. Townsend, PhD, Clinical Imaging Research Centre, A*STAR-NUS Singapore

- A. Mark Richards, MBChB, MD, PhD, DSc, FRACP, FRCP, FRSNZ, Cardiovascular Research Institute, NUHS, Singapore

- John J. Totman, DCR, MSc, PhD, Clinical Imaging Research Centre, ASTAR Singapore

- Mark Y.Y. Chan, MBBS, MHS, MMED, MRCP, Department of Cardiology, National University Heart Centre, Singapore

1. From 15 August 2016 Therese Sjoholm works at the Department of Radiology, Uppsala University, Sweden

Corresponding address:

Stephanie Marchesseau, National University of Singapore, 14 medical drive, \#B1-01, Singapore 117599, Tel: 6601 3525, marchesseau.stephanie@gmail.com

Funding: This work has been partially funded by the NMRC NUHS Centre Grant - Medical Image Analysis Core (NMRC/CG/013/2013). 


\section{ABSTRACT}

Background-Following an acute coronary syndrome, combined CT and PET with ${ }^{18} \mathrm{~F}-\mathrm{NaF}$ can identify coronary atherosclerotic plaques that have ruptured or eroded. However, the processes behind ${ }^{18} \mathrm{~F}-\mathrm{NaF}$ uptake in vulnerable plaques remain unclear.

Methods and Results- Ten patients with STEMI were scanned after ${ }^{18} \mathrm{~F}-\mathrm{NaF}$ injection, for 75 minutes in a Siemens PET/MR scanner using delayed enhancement (LGE). They were then scanned in a Siemens PET/CT scanner for 10 minutes. Tissue-To-Background ratio (TBR) was compared between the culprit lesion in the IRA and remote non-culprit lesions in an effort to independently validate prior studies. Additionally, we performed a proof-of-principle study comparing TBR in scar tissue and remote myocardium using LGE images and PET/MR or PET/CT data. From the 33 coronary lesions detected on PET/CT, TBRs for culprit lesions were higher than for non-culprit lesions ( $\mathrm{TBR}=2.11+/-0.45$ versus $1.46+/-0.48 ; \mathrm{P}<0.001$ ). Interestingly, the TBR measured on the PET/CT was higher for infarcted myocardium than for remote myocardium $(\mathrm{TBR}=0.81+/-0.10$ versus $0.71+/-0.05 ; \mathrm{P}=0.003)$. These results were confirmed using the $\mathrm{PET} / \mathrm{MR}$ data $(\mathrm{TBR}=0.81+/-0.10$ for scar, $\mathrm{TBR}=0.71+/-0.06$ for healthy myocardium, $\mathrm{P}=0.03)$.

Conclusions - We confirmed the potential of ${ }^{18} \mathrm{~F}-\mathrm{NaF}$ PET/CT imaging to detect vulnerable coronary lesions. Moreover, we demonstrated proof-of-principle that ${ }^{18} \mathrm{~F}-\mathrm{NaF}$ concurrently detects myocardial scar tissue.

\section{ABBREVIATIONS}

CT: Computed Tomography

PET: Positron Emission Tomography

STEMI: ST- elevation Myocardial Infarction

IRA: infarct-related artery

TBR: Tissue-To-Background ratio

LGE: Late Gadolinium Enhancement (imaging)

${ }^{18} \mathrm{~F}-\mathrm{NaF}$ : sodium fluoride tracer 


\section{INTRODUCTION}

Acute myocardial infarction (AMI) is a leading cause of death and disability worldwide. AMI occurs when rupture or erosion of coronary atheroma leads to thrombus formation and sudden occlusion of a major epicardial coronary artery[1]. While coronary atheromata are commonly present in many middle aged and older adults, certain atheromatous plaques are more susceptible to rupture and erosion. Termed 'vulnerable plaques', these atheromata are histologically defined by a large lipid rich core and thin overlying fibrous cap[2,3]. The medical imaging community has recognized that mere identification of atheromatous plaques[4] is insufficient as many atheromatous plaques are stable and not prone to rupture or erosion[5,6]. It is therefore of great interest to the community to develop tools to better identify vulnerable plaques as the presence of these plaques may indicate an increased risk of AMI, therefore prompting escalation of medical treatment or targeted mechanical stabilization of vulnerable plaques by selective coronary stenting. Many modalities presently developed to identify vulnerable coronary plaques are invasive in nature, requiring the insertion of physical devices into a patient's coronary arteries[7, 8]. Despite the superior spatiotemporal resolution of these invasive modalities, they carry a small but definite risk of causing coronary artery injury and AMI. As such, non-invasive modalities to identify vulnerable coronary plaques are a key unmet need in coronary artery imaging. Recently, noninvasive PET-CT has been used to identify high-risk plaques using ${ }^{18} \mathrm{~F}-\mathrm{NaF}[9,10]$. Joshi et al.[11] showed that the Tissue-to-Background ratio (TBR) for culprit plaques was significantly higher than for non-culprit plaques (1.66 vs 1.24) on ${ }^{18} \mathrm{~F}-\mathrm{NaF}$ PET/CT imaging of 40 patients with recent myocardial infarction.

It is presently unclear if ${ }^{18} \mathrm{~F}-\mathrm{NaF}$ has other potential uses in cardiac imaging. While it is acknowledged that ${ }^{18} \mathrm{~F}-\mathrm{NaF}$ can detect culprit coronary atheromata, the mechanism behind this detection remains unclear. More recently, Irkle et al.[12] demonstrated preferential binding of ${ }^{18} \mathrm{~F}$ $\mathrm{NaF}$ to microcalcification. As prior studies have shown varying degrees of calcification in previously infarcted myocardium, we hypothesized that ${ }^{18} \mathrm{~F}-\mathrm{NaF}$ could detect scar tissue arising from myocardial infarction. Moreover, ${ }^{18} \mathrm{~F}-\mathrm{NaF}$ may possibly target apoptosis, as demonstrated in rat imaging[13], or macrophage infiltration and necrosis, as suggested by histological studies of carotid artery plaques [11], processes that are common to both atherosclerotic plaque formation and myocardial infarction. We therefore sought first to independently validate the use of ${ }^{18} \mathrm{~F}-\mathrm{NaF}$ PET/CT imaging for the detection of high-risk coronary atheromata. Using ${ }^{18} \mathrm{~F}-\mathrm{NaF}$ PET/MR imaging, we then sought to quantify the uptake of ${ }^{18} \mathrm{~F}-\mathrm{NaF}$ in myocardial scar tissue relative to remote healthy myocardium to add understanding of the common processes between infarction and atheromata.

\section{MATERIALS and METHODS}

\section{Study population}

For this pilot study, we enrolled 10 consecutive patients presenting with STEMI undergoing primary (emergency) percutaneous coronary intervention (PCI) at the National University Heart Centre Singapore. STEMI was determined by a typical history of ischemic chest pain or angina equivalent symptoms, new ST elevation at the $\mathrm{J}$ point in at least 2 contiguous leads of $\geq 2 \mathrm{~mm}(0.2$ $\mathrm{mV})$ in men or $\geq 1.5 \mathrm{~mm}(0.15 \mathrm{mV})$ in women in leads $\mathrm{V} 2-\mathrm{V} 3$ and/or of $\geq 1 \mathrm{~mm}(0.1 \mathrm{mV})$ in other contiguous chest leads or the limb leads, accompanied by a typical rise and/or fall of cardiac troponin[14]. All patients had an acute occlusion of an infarct-related artery (IRA) on coronary angiography and underwent emergency PCI with stent placement within 12 hours of symptom 
onset. After PCI, consented patients were screened for eligibility and were then scanned at a median of 13.5 days (range 9-24) after the acute event on the PET/MR followed immediately by PET/CT.

We excluded patients with MR contraindications such as metallic implants, pacemakers or claustrophobia. Additionally, we excluded patients with severe renal and liver impairment, significant valvular heart disease, and a history of malignancy.

The study was approved by the National Healthcare Group Domain Specific Review Board (DSRB reference number 2013/00248) and written informed consent was obtained from all patients.

\section{PET/MR image acquisition and reconstruction}

All subjects were first scanned on a Biograph mMR PET-MR scanner (Siemens Healthineers, Erlangen, Germany) for 75 minutes. MRI and PET scans were performed simultaneously on a single bed centered over the heart. The PET-MRI scan was started immediately after intravenous injection of $2.95+/-0.21 \mathrm{mCi} 18 \mathrm{~F}-\mathrm{NaF}$. PET data was reconstructed from the last 15 minutes (6075 min after injection) using an iterative reconstruction (Siemens OP-OSEM algorithm, 3 iterations, 21 subsets, matrix size 344x344 with Point Spread Function[15] correction) without ECG or respiratory gating to decrease the level of noise. The UMAP was created from a Dixon MRI sequence acquired at the beginning of the PET recording. The reconstructed images were smoothed with a $2 \mathrm{~mm}$ FWHM Gaussian filter, matching the voxel dimension of $2 \times 2 \times 2 \mathrm{~mm}$. $18 \mathrm{~F}-\mathrm{NaF}$ uptake in the scar and remote myocardium was quantified by the standardized uptake value (SUV).

\section{Cardiovascular Magnetic Resonance (CMR) Protocol and Measurements}

Image Acquisition:

In addition to the localizer, the MRI cardiac imaging protocol included a breath-hold CINE steady state free precession (SSFP) short axis view covering both the right ventricle (RV) and the left ventricle (LV). Delayed enhancement (LGE) short axis images were acquired 10 minutes after a $0.2 \mathrm{mmol} / \mathrm{kg}$ GADOVIST injection. Sequential parameters of the LGE acquisition were the following: $\mathrm{TR}=4.1 \mathrm{~ms}$, Average $\mathrm{R}-\mathrm{R}$ interval $=990 \mathrm{~ms}, \mathrm{TE}=1.56 \mathrm{~ms}$, Slice thickness $=8 \mathrm{~mm}$, $\mathrm{Gap}=20 \%, \mathrm{FOV}=276 \times 340 \mathrm{~mm}$, Matrix $=133 \times 256$, Flip angle=20, Inversion time=subjectspecific calculated using TI scout, Image resolution=1.33 x $1.33 \times 10 \mathrm{~mm}$.

Image Analysis:

All CMR sequences were analyzed jointly by 2 trained image analysts using the 510k FDA cleared software Segment version 1.9 R3556[16]. Left Ventricular (LV) segmentation of the endocardium and epicardium was performed automatically on all timeframes of the short-axis CINE stack. Manual adjustment of the contours was performed if necessary, following the SCMR guidelines[17]. In addition, LGE images were analyzed by delineating the LV endocardium and epicardium manually and extracting the scar tissue automatically using Otsu's threshold[18].

\section{PET/CT image acquisition and reconstruction}

Of the 10 subjects who underwent PET/MR scanning, two subjects declined subsequent PET/CT scanning. The remaining eight subjects were then immediately transferred to a Biograph mCT 
PET/CT scanner (Siemens Healthineers, Erlangen, Germany). A non-enhanced coronary artery calcium score CT scan $(120 \mathrm{kV}, 120$ quality reference mAs, CareDose, $300 \mathrm{~ms}$ exposure time, $128 \times 0.6 \mathrm{~mm}$ collimation, non-triggered acquisition) and a low dose CT attenuation correction scan were first performed using the 128-row CT scanner in the PET/CT system. The calcium scan was reconstructed at a slice thickness of $3 \mathrm{~mm}$ using an iterative reconstruction (Siemens SAFIRE) to allow high resolution imaging of the coronary arteries.

The CT scans were immediately followed by a 10 min PET scan (one bed position centered over the heart). The scan time was limited to 10 minutes to ease the protocol for the patients who previously underwent 75 minutes of PET/MRI scanning. The PET scan was started at least 90 min post ${ }^{18} \mathrm{~F}-\mathrm{NaF}$ injection (108 +/- $\left.21 \mathrm{~min}\right)$ and was reconstructed using an iterative reconstruction (Siemens OP-OSEM algorithm, 3 iterations, 21 subsets, $2 \mathrm{~mm}$ pixel size, 2mm FWHM Gaussian filter), with attenuation and scatter corrections included as well as Time of Flight[19] and Point Spread Function[15] correction. Although the PET scan was acquired using respiratory and cardiac gating, the gated information was not used in the reconstruction as it resulted in images with suboptimal count statistics.

\section{Tissue-To-Background ratios calculation}

Coronary atheromata TBR from PET/CT:

Calcium regions within the arteries were delineated manually on the CT image, having higher intensities than surrounding blood as shown Figure 1. Additionally, each vessel containing at least one calcium region was partly segmented manually from axial slices of the 3D CT image. Hybrid PET-CT acquisition ensures the direct transposition of these Regions of Interest (ROIs) on the PET image, therefore enabling the automatic measurement of SUV within each region. This direct transposition of the ROIs does not guarantee an optimal quantification of SUV due to the rapid motion and small size of the atheromata as it has been demonstrated before[20]. Since a consensus on solving these misregistration problems has not been validated yet, the authors decided to use a simple approach and measure the TBR of each calcium ROI as already validated by Josh et al.[11]. It is defined as the ratio between the calcium region maximum SUV over the corresponding vessel (excluding the calcium ROIs) mean SUV:

$$
T B R_{\text {atheromata }}(R O I)=\frac{S U V_{\max }(R O I)}{S U V_{\text {mean }}(\text { vessel })}
$$

Myocardial infarct TBR from PET/MR:

In order to correct possible misalignment between the LGE images acquired half way through the CMR protocol on 10-15 breath-holds, and the PET/MR image acquired continuously over 15 minutes towards the end of the CMR protocol, a first automatic registration was performed. As described before, LGE short-axis images were first analyzed in order to extract the scar regions and the healthy myocardium regions. The images and resulting masks were then resampled to an isotropic resolution $(1.33 \times 1.33 \times 1.33 \mathrm{~mm})$ for a better fit with the PET image. As described Fig. 2 (left), the Dixon (in-phase) image acquired along the PET is first resampled to the same short-axis acquisition plane as the LGE image to ease the registration between the two images. An in-house algorithm (developed using ITK libraries) using well validated mutual information metric[21] to perform the rigid 3D registration was used. Despite the different image intensities in both images (the myocardium is not visible on Dixon), the registration was successful as Fig.2 (left).

After automatic realignment of LGE and Dixon, registered scar and remote healthy myocardium 
ROIs were reported directly on the Dixon (for verification) and the PET image for SUV quantification. The TBR in the myocardium was defined differently from the TBR in the atheromata, as the mean SUV within the ROI (scar or remote myocardium) divided by the mean SUV within the left ventricle blood pool away from the walls:

$$
T B R_{\text {myocardium }}(R O I)=\frac{S U V_{\text {mean }}(R O I)}{S U V_{\text {mean }}(L V \text { blood pool })}
$$

The mean SUV of the ROI was considered instead of the max SUV to account for the large scar and remote myocardium regions compared to the atheromata ROIs. Although the blood pool may show local turbulence, the averaged value of blood pool SUV acquired over 15 minutes of PET acquisition was chosen to normalize the infarct (and remote myocardium) uptake since it is assumed to be consistent across patients who underwent an identical protocol.

\section{Myocardial infarct TBR from PET/CT:}

As the PET data acquired on PET/CT were of higher quality than the PET-MRI data, myocardial infarct TBRs were also measured on the PET/CT to confirm the results. As described Fig.2 (right), the LGE MRI images had first to be aligned to the PET/CT images. First the CT images were resampled on the scanner software Siemens SAFIRE in order to obtain short-axis views (2mm thickness), and 2 chamber and 4 chamber views corresponding to the MRI LGE image orientation. Then, MedINRIA 1.9[22] image fusion software was used for a manual landmark based rigid registration to align the isoresampled LGE image and masks (obtained from the LGE to PET/MR registration step) to the resampled $\mathrm{CT}$ image chosen as the reference image. The junctions between outflow and inflow vessels were used as landmarks for the co-registration as well as the apex tip. Similarly to the PET/MR registration, the resulting transformation was then applied to deform the myocardium and scar ROIs. Fig. 2 (right) shows an example of the final alignment of the segmentations on CT (for verification) and PET images, with good agreement despite the lack of muscle landmark on the non-contrast CT. After registration, registered scar ROIs and remote healthy myocardium were reported directly on the PET image and SUV and TBR values extracted identically to the PET/MR method.

\section{Angiography examination}

Patients underwent emergent coronary angiography and primary percutaneous coronary intervention for STEMI via transradial or transfemoral access. Coronary angiograms were acquired on a Siemens biplane system Artis system (Siemens, Munich, Germany) using standardized tomographic views of right anterior oblique caudal, anterior posterior cranial, left anterior oblique cranial and left anterior oblique caudal for the left coronary system, and left anterior oblique cranial and right anterior oblique for the right coronary system. The volume of contrast (Omnipaque) used for each coronary angiogram was typically between 25-50 ml. All patients underwent manual thrombectomy, balloon angioplasty and coronary stent implantation as directed by the primary operator.

Culprit plaques were spatially defined by the presence of an occlusive or subocclusive lesion within the IRA corresponding to electrocardiographic and echocardiographic localization. Culprit lesions on coronary angiography were then mapped to corresponding calcium regions on the CT image and each calcium region assigned a culprit or non-culprit label. 


\section{Statistical analysis}

Coronary atheromata:

The TBR of identified culprit and non-culprit lesions were compared statistically using a 2-tailed Student's t-test and the mean and standard deviation (SD) for culprit versus non-culprit plaques recorded. The effect size (d) using Cohen's definition (difference of the group means divided by the average standard deviation) was also calculated in order to judge the magnitude of the difference $(\mathrm{d} \geq 0.8$ is considered a large difference[23]). From the mean and standard deviation of the culprit and non-culprit plaque TBR, 95\% confidence intervals (CI) were computed (mean+/1.96SD). Using these non-overlapping intervals, a cutoff-value was defined as the mean between the lower bound of the $95 \%$ CI of culprit plaques and the higher bound of the $95 \%$ CI of nonculprit plaques. Sensitivity and specificity values to classify high and low-risk plaques with this cutoff value were then calculated.

\section{Myocardial infarct:}

TBR between scar and remote myocardium were compared statistically using a 2-tailed Student's t-test and Cohen's effect size to account for the small sample size. Statistical software package in Matlab R2013b was used for all analyses and a 2-sided $\alpha$ of 5\% was considered statistically significant. A Pearson's correlation was also established between the PET/MR and PET/CT SUVs.

\section{RESULTS}

\section{Baseline characteristics}

The cohort comprised of 9 men and 1 woman with a mean age of $48 \pm 7$ years. All received a loading dose of aspirin and ADP-receptor inhibitor before the coronary intervention and had a median door-to-balloon time of 42 minutes (interquartile range 24). Median peak Troponin I was 69.5ng/ml (interquartile range 45.6), indicating significant myocardial damage. Two subjects declined to undergo PET/CT after the first PET/MRI scan. Baseline and CMR characteristics are shown in Table 1.

\section{Visual analysis}

\section{Coronary atheromata:}

Atheromatous plaques were segmented on the CT calcium scoring image as regions of high intensity (white) within the coronaries (see Fig.1c). Hybrid PET/CT imaging allowed automatic co-registration of the coronary anatomy and the PET image. This can however be imperfect due to patient, respiratory and cardiac motions. Visual distinction of vulnerable plaques was challenging due to the small size of the plaques, the limited resolution of the PET image, as well as respiratory and cardiac motion, leading to high partial volume effect (see Fig.1d). In the example shown in Figure 1, the culprit (high risk) plaque as assessed by angiography corresponds to ROI1 while ROI2 was diagnosed as a low risk plaque. On the PET image, a visual difference in uptake between the two ROIs is noticeable, however, the maximal SUV of the culprit plaque is of the same order of magnitude as the noise in the surrounding blood. This non-specific uptake renders the detection of vulnerable plaques impossible if SUVs of plaques are compared without normalization to background. 
Myocardial scar:

Report of the myocardium and scar ROIs after registration of the LGE image and the PET/CT was visually successful (as assessed by two independent observers) on all cases allowing confidence in the measurements. Similarly, the automatic registration of LGE and Dixon (to align with PET/MR) was necessary for 2 of the 10 cases and allowed to correct for patient motion. The other 8 patients showed more stillness during the scan leading to already aligned images (as assessed by two independent observers) for which the automatic registration showed no improvement.

Visual distinction of scar tissue on the PET images (both PET/MR and PET/CT) was as challenging as for the atheromata. An example is shown Fig.2 (left for PET/MR and right for PET/CT on the same patient) in which most parts of the scar have higher uptake than the average myocardium. However, partial volume effect, breathing and cardiac beating reduce the uptake to a low value. One can also notice that the uptake in the blood pool is globally higher than in the myocardium. Once again the uptake is not specific to the scar and similar uptake is observed in the turbulent blood pool or surrounding tissue. For a better comparison of regional muscle uptake, PET images were masked to the full myocardium and overlaid on LGE images as shown Fig.3. Clear distinction can be made between healthy and infarct uptake. However, as one can notice, the uptake is not homogeneous within the scar region.

\section{Parametric comparison}

Coronary atheromata:

From the 33 detected lesions on CT, 8 were identified as being culprit on the standard coronary angiogram, 1 for each patient undergoing PET/CT. The TBR of the culprit lesions was found significantly higher than the TBR for non-culprit lesions (Fig.4 left) with an average value of 2.11 for culprit $( \pm 0.42)$ and $1.36( \pm 0.30)$ for non-culprit (p-value $<0.001, d=1.77)$. Considering only non-overlapping confidence intervals, a threshold for the classification of high-risk and low-risk lesions was defined at 1.70 . This led to a specificity of $72 \%$, a sensitivity of $87.5 \%$ and an accuracy of $75.7 \%$ for detecting culprit coronary atheromata in these 8 patients.

Myocardial scar:

Nine of the 10 patients had clear visible zones of scar tissue defined on the LGE image. In the patient without clear LGE, the IRA was the right posterior descending artery (RPDA), which is the likely reason for the absence of definite scar. Scar percentages are reported in Table 3 along with the TBR and SUV for scar relative to remote healthy myocardium for each patient and each image modality. The TBR was found to be significantly higher in scar tissue than in the remote healthy myocardium with an average value of $0.87( \pm 0.10)$ for scar and $0.72( \pm 0.05)$ for remote myocardium ( $\mathrm{p}$-value $=0.003, \mathrm{~d}=1.86$ ) on the PET/CT for the 8 patients (Fig.4 right). Similarly, despite lower image quality of the PET/MR, the TBR was also found significantly higher in scar tissue $(\mathrm{TBR}=0.81 \pm 0.10)$ than in remote myocardium $(\mathrm{TBR}=0.72 \pm 0.06),(\mathrm{p}-\mathrm{value}=0.03, \mathrm{~d}=1.55)$ for the 10 patients. Additionally, including only the 8 patients that underwent both PET/CT and PET/MR, SUV measured with PET/CT and PET/MR showed significant Pearson's correlations of 0.75 for scar and 0.71 for remote myocardium despite the small number of patients. From the PET/MR data, there was no significant correlation between the size of the scar tissue and the uptake ( $\mathrm{p}$-value $=0.91, \mathrm{R}=-0.04$ between scar size and TBR scar, $\mathrm{p}$-value $=0.33, \mathrm{R}=-0.36$ between scar size and SUV scar) consistent with the measure being averaged on the scar zone. There was 
also no correlation between the scar size or the uptake with respect to the PCI-to-Scan time (pvalue $=0.28, \mathrm{R}=-0.40$ between TBR scar and PCI-to-Scan time; $\mathrm{p}$-value $=0.33, \mathrm{R}=-0.36$ between SUV scar and PCI-to-Scan time; $\mathrm{p}$-value $=0.34, \mathrm{R}=-0.35$ between scar size and PCI-to-Scan time).

\section{DISCUSSION}

Our study results first confirm previous findings of successful ${ }^{18} \mathrm{~F}-\mathrm{NaF}$ differentiation of culprit coronary atheromata in the IRA of patients with recent STEMI, despite noisy PET images and a non-optimal protocol that do not allow direct visual assessment. As shown, and contrary to Joshi et al.[11], a distinct identification of culprit plaques on the PET images was hardly noticed in our study despite the highly significant TBR differences. This difference may be due to respiratory and cardiac motion, small size of the lesions leading to high partial volume effect or insufficient injected activity. A longer scan time would have allowed ECG and respiratory gating, probably leading to more accurate results, less partial volume effect and improved image quality. Additionally, new reconstruction algorithms can be thought of to reduce the noise level (for instance, the work by Rubeaux et al.[20]). Nevertheless, the amount of noise from all these factors affects the TBR of all plaques, making the culprit vs non-culprit comparison relevant. In addition, this pilot study included a small cohort of patients as an initial proof of concept of concomitant coronary and myocardial $18 \mathrm{~F}-\mathrm{NaF}$ PET imaging. Despite the limitations of our protocol, the limited study size and the unspecific uptake, the difference in TBR were highly significant proving once more the potential of such an approach to differentiate between atheromata. As for all other similar studies, patients were scanned after PCI which is likely to have affected the plaque prior to scanning. Joshi et al.' histological validation[11] indicates nonetheless that this is not a major limitation. The accuracy of only $76 \%$, however, tends to confirm previous skepticism on the clinical value of the method since more than one plaque might have ruptured unnoticed[8]. It is therefore clear that more research is still required before $18 \mathrm{~F}-\mathrm{NaF}$ PET imaging can be used as a diagnostic tool and more validation needed in order to establish a PET threshold to define a vulnerable plaque. For now, as described in the "myth of the vulnerable plaque" by Arbab-Zadeh and Fuster[24], a more sensible approach will be to treat systemic risk rather than focus on specific high risk plaques.

The main contribution of this study was to identify, for the first time in humans, simultaneous ${ }^{18} \mathrm{~F}$ $\mathrm{NaF}$ uptake in myocardial scar in the territory supplied by the IRA using both PET/CT after registration with LGE and PET/MR. Co-registration with CMR enabled structural myocardial information otherwise unavailable from the CT images to be incorporated into the PET/CT analysis and directly compared with the PET/MR analysis. Despite spillage from the left ventricular blood pool that could affect the SUV for both myocardial scar (mainly transmural in our study) and remote myocardium, partial volume effect due to respiratory and cardiac motion, and potential misregistration between non-contrast CT and MRI and between PET/MR and LGE, we demonstrated twice that ${ }^{18} \mathrm{~F}-\mathrm{NaF}$ is taken up by both culprit coronary atheromata and myocardial scar tissue. PET/MRI acquisition does not include Time of Flight, and the reconstruction is based on a UMAP of lower accuracy than what is obtained from a CT scan. Despite these limitations, the results correlated with the PET/CT results which strengthens our hypothesis that ${ }^{18} \mathrm{~F}-\mathrm{NaF}$ targets myocardial scar significantly. Additionally, the non-homogenous uptake of ${ }^{18} \mathrm{~F}-\mathrm{NaF}$ in the myocardial infarct seems to indicate various processes occurring in postMI regions that LGE MRI does not report on. Further studies are warranted to characterize these processes and evaluate the complementarity of ${ }^{18} \mathrm{~F}-\mathrm{NaF}$ PET with other scar imaging techniques 
as our data suggest that ${ }^{18} \mathrm{~F}-\mathrm{NaF}$ imaging reports on biological processes beyond that elucidated by LGE MRI.

Previous studies intended to explain why ${ }^{18} \mathrm{~F}-\mathrm{NaF}$ binds to ruptured or vulnerable plaque offer the following hypothesis: atherosclerotic vascular calcification is a cellular response to necrotic and chronic inflammatory stimulus[11], therefore directly linked to inflammation. Additionally, fluoride ions are incorporated in the process of microcalcification[5, 6]. Recently Irkle et al.[12] differentiated stable macrocalcification from high risk microcalcification therefore explaining the preferred binding of ${ }^{18} \mathrm{~F}-\mathrm{NaF}$ to culprit plaques. Moreover, Irkle et al.[12] proved that this specific uptake in the culprit plaques did not correlate with any markers of inflammation which therefore excludes the hypothesis that ${ }^{18} \mathrm{~F}-\mathrm{NaF}$ targets inflammation resulting from stent injury in the artery or any other inflammation processes.

Studies have identified calcification of infarcted myocardium on CT images and in postmortem examinations[25]. In canine models of AMI, permanent coronary ligation did not increase the uptake of radio-labelled $45 \mathrm{Ca}-\mathrm{CaCl}$ but ischemia followed by reperfusion led to an 18 -fold increase in calcium uptake in the injured myocardium[26,27]. Calcium uptake is postulated to be an active process associated with mitochondrial accumulation of calcium into dense intramitochondrial granules of calcium phosphate. Mitophagy of cardiomyocytes, is often triggered by massive calcium influx into cardiomyocyte mitochondria exposed to ischemiareperfusion injury. The uptake of mitochondrial is a feature of irreversible cellular injury and may be related to defects in mitochondrial membrane permeability. Moreover, increased uptake calcium could also be due to retention of calcific deposits in the extracellular space present due to myocyte death or from the myocyte necrosis process itself[28]. Our study did not include histological findings to determine with certainty the common processes between myocardial infarct and culprit atheromata uptake but we hypothesize that many of the cell death mechanisms in atherosclerotic plaque disruption are common to myocardium exposed to ischemia-reperfusion injury.

\section{New Knowledge Gained}

The use of ${ }^{18} \mathrm{~F}-\mathrm{NaF}$ PET/CT imaging to detect high-risk coronary lesions in patients with STEMI is confirmed in a small Asian population. The comparison of ${ }^{18} \mathrm{~F}-\mathrm{NaF}$ uptake in myocardial scar tissue with remote healthy myocardium shows, for the first time in humans, that ${ }^{18} \mathrm{~F}-\mathrm{NaF}$ also binds to myocardial scar tissue.

These data serve as proof-of-principle that first, pathobiological processes involving microcalcification may be common to both atherosclerotic plaque disruption and myocardial infarction and second, ${ }^{18} \mathrm{~F}-\mathrm{NaF}$ PET/CT could be potentially applied to simultaneous coronary and myocardial imaging applications to quantify both vulnerable plaque burden and myocardial scar tissue.

\section{Compliance with Ethical Standards}

Ethical approval: All procedures performed in studies involving human participants were in accordance with the ethical standards of the institutional and/or national research committee and with the 1964 Helsinki declaration and its later amendments or comparable ethical standards. This article does not contain any studies with animals performed by any of the authors. 
Informed consent: Informed consent was obtained from all individual participants included in the study.

Conflict of Interest: Stephanie Marchesseau, Aruni Seneviratna, A. Therese Sjoholm, Daphne Liang Qin, Jamie X.M. Ho, Derek J. Hausenloy, David W. Townsend, A. Mark Richards, John J. Totman and Mark Y.Y. Chan declare that they have no conflict of interest.

\section{REFERENCES}

1. Davies MJ. The pathophysiology of acute coronary syndromes. Heart. 2000;83:361-6.

2. Kolodgie FD, Burke AP, Farb A, Gold HK, Yuan J, Narula J, et al. The thin-cap fibroatheroma: a type of vulnerable plaque: the major precursor lesion to acute coronary syndromes. Curr Opin Cardiol. 2001;16:285-92.

3. Naghavi M, Libby P, Falk E, Casscells SW, Litovsky S, Rumberger J, et al. From vulnerable plaque to vulnerable patient a call for new definitions and risk assessment strategies: Part II. Circulation. 2003;108:1772-8.

4. Budoff MJ, Gul KM. Expert review on coronary calcium. Vascular health and risk management. 2008;4:315.

5. Huang H, Virmani R, Younis H, Burke AP, Kamm RD, Lee RT. The impact of calcification on the biomechanical stability of atherosclerotic plaques. Circulation. 2001;103:1051-6.

6. Vengrenyuk Y, Carlier S, Xanthos S, Cardoso L, Ganatos P, Virmani R, et al. A hypothesis for vulnerable plaque rupture due to stress-induced debonding around cellular microcalcifications in thin fibrous caps. Proceedings of the National Academy of Sciences. 2006;103:14678-83.

7. Maehara A, Mintz GS, Bui AB, Walter OR, Castagna MT, Canos D, et al. Morphologic and angiographic features of coronary plaque rupture detected by intravascular ultrasound. J Am Coll Cardiol. 2002;40:904-10.

8. Rioufol G, Finet G, Ginon I, Andre-Fouet X, Rossi R, Vialle E, et al. Multiple atherosclerotic plaque rupture in acute coronary syndrome a three-vessel intravascular ultrasound study. Circulation. 2002;106:804-8.

9. Dweck MR, Jones C, Joshi NV, Fletcher AM, Richardson H, White A, et al. Assessment of valvular calcification and inflammation by positron emission tomography in patients with aortic stenosis. Circulation. 2012;125:76-86.

10. Dweck MR, Chow MW, Joshi NV, Williams MC, Jones C, Fletcher AM, et al. Coronary arterial 18F-sodium fluoride uptake: a novel marker of plaque biology. J Am Coll Cardiol. 2012;59:1539-48.

11. Joshi NV, Vesey AT, Williams MC, Shah AS, Calvert PA, Craighead FH, et al. 18 F-fluoride positron emission tomography for identification of ruptured and high-risk coronary atherosclerotic plaques: a prospective clinical trial. The Lancet. 2014;383:705-13.

12. Irkle A, Vesey AT, Lewis DY, Skepper JN, Bird JL, Dweck MR, et al. Identifying active vascular microcalcification by $18 \mathrm{~F}$-sodium fluoride positron emission tomography. Nature communications. 2015;6.

13. Han JH, Lim SY, Lee MS, Lee WW. Sodium [18F] Fluoride PET/CT in Myocardial Infarction. Mol Imaging Biol. 2015;17:214-21.

14. Antman EM, Anbe DT, Armstrong PW, Bates ER, Green LA, Hand M, et al. ACC/AHA guidelines for the management of patients with ST-elevation myocardial infarction-executive summary: a report of the American College of Cardiology/American Heart Association Task Force on Practice Guidelines (Writing Committee to Revise the 1999 Guidelines for the Management of Patients With Acute Myocardial Infarction). J Am Coll Cardiol. 2004;44:671-719.

15. Panin VY, Kehren F, Michel C, Casey M. Fully 3-D PET reconstruction with system matrix derived from point source measurements. IEEE Trans Med Imaging. 2006;25:907-21.

16. Heiberg E, Sjögren J, Ugander M, Carlsson M, Engblom H, Arheden H. Design and validation of Segmentfreely available software for cardiovascular image analysis. BMC Med Imaging. 2010;10:1.

17. Schulz-Menger J, Bluemke DA, Bremerich J, Flamm SD, Fogel MA, Friedrich MG, et al. Standardized image interpretation and post processing in cardiovascular magnetic resonance: Society for Cardiovascular Magnetic Resonance (SCMR) board of trustees task force on standardized post processing. J Cardiovasc Magn Reson. 2013;15:1.

18. Heiberg E, Engblom H, Engvall J, Hedström E, Ugander M, Arheden H. Semi-automatic quantification of myocardial infarction from delayed contrast enhanced magnetic resonance imaging. Scand Cardiovasc J. 2005;39:26775 . 
19. Conti M, Bendriem B, Casey M, Chen M, Kehren F, Michel C, et al. First experimental results of time-offlight reconstruction on an LSO PET scanner. Phys Med Biol. 2005;50:4507.

20. Rubeaux M, Joshi NV, Dweck MR, Fletcher A, Motwani M, Thomson LE, et al. Motion correction of 18FNaF PET for imaging coronary atherosclerotic plaques. J Nucl Med. 2016;57:54-9.

21. Wells WM, Viola P, Atsumi H, Nakajima S, Kikinis R. Multi-modal volume registration by maximization of mutual information. Med Image Anal. 1996;1:35-51.

22. Toussaint N, Souplet J-C, Fillard P. MedINRIA: medical image navigation and research tool by INRIA. Proc of MICCAI: Citeseer; 2007. p. 280.

23. Sullivan GM, Feinn R. Using effect size-or why the P value is not enough. J Grad Med Educ. 2012;4:279-

82.

24. Arbab-Zadeh A, Fuster V. The myth of the "vulnerable plaque": transitioning from a focus on individual lesions to atherosclerotic disease burden for coronary artery disease risk assessment. J Am Coll Cardiol. 2015;65:84655.

25. Shriki JE, Shinbane J, Lee C, Khan AR, Burns N, Hindoyan A, et al. Incidental myocardial infarct on conventional nongated CT: a review of the spectrum of findings with gated CT and cardiac MRI correlation. American Journal of Roentgenology. 2012;198:496-504.

26. Shen AC, Jennings RB. Kinetics of calcium accumulation in acute myocardial ischemic injury. The American journal of pathology. 1972;67:441.

27. D'Agostino AN, Chiga M. Mitochondrial Mineralization in Human Myocardium. Am J Clin Pathol. 1970;53:820-4. doi:10.1093/ajcp/53.6.820.

28. Dongworth RK, Hall AR, Burke N, Hausenloy DJ. Targeting mitochondria for cardioprotection: examining the benefit for patients. Future Cardiol. 2014;10:255-72. 


\section{Tables}

\begin{tabular}{|c|c|c|}
\hline & PET/MR & $+\mathrm{PET} / \mathrm{CT}$ \\
\hline Population Size, $n$ & 10 & 8 \\
\hline Age, yrs (SD) & $48.2(7.4)$ & $46.9(7.7)$ \\
\hline Male, n (\%) & $9(100)$ & $8(100)$ \\
\hline Hypertension, n (\%) & $2(20)$ & $1(12.5)$ \\
\hline Dyslipidemia, n (\%) & $1(10)$ & $1(12.5)$ \\
\hline Current Smoker, n (\%) & $3(30)$ & $3(37.5)$ \\
\hline DAPT at discharge, n (\%) & $10(100)$ & $8(100)$ \\
\hline $\begin{array}{l}\text { LV ejection fraction, \% } \\
\text { (SD) }\end{array}$ & $62.3(6.7)$ & $59.9(4.8)$ \\
\hline LV mass, g (SD) & $139.6(22.5)$ & $140.6(25.4)$ \\
\hline $\begin{array}{l}\text { LV end-diastolic volume, } \mathrm{ml} \\
\text { (SD) }\end{array}$ & $121.9(10.9)$ & $122.8(11.3)$ \\
\hline $\begin{array}{l}\text { LV end-systolic volume, } \mathrm{ml} \\
\text { (SD) }\end{array}$ & $46.2(10.5)$ & $49.5(8.8)$ \\
\hline LV stroke volume, ml (SD) & $75.8(8.5)$ & $73.3(6.5)$ \\
\hline $\begin{array}{l}\text { Presence of scar tissue, } n \\
(\%)\end{array}$ & $9(90.0)$ & $7(87.5)$ \\
\hline $\begin{array}{l}\text { Peak Troponin I level, ng/ml } \\
\text { (SD) }\end{array}$ & $48.5(30.4)$ & $55.9(29.0)$ \\
\hline PCI to Scan time (days, SD) & $15.1(4.5)$ & $15.5(5)$ \\
\hline
\end{tabular}


Table 2. Per patient comparison of the culprit and non-culprit Tissue-to-Background ratio

\begin{tabular}{lllllllllll}
\hline Patients & $\mathbf{1}$ & $\mathbf{2}$ & $\mathbf{3}$ & $\mathbf{4}$ & $\mathbf{5}$ & $\mathbf{6}$ & $\mathbf{7}$ & $\mathbf{8}$ & $\begin{array}{l}\text { Mean } \\
\text { (SD) }\end{array}$ \\
$\begin{array}{l}\text { Position of } \\
\text { rupture }\end{array}$ & RPDA & LAD & LAD & LAD & LAD & LAD & LAD & RCA & \\
$\begin{array}{l}\text { Num. of } \\
\text { ROIs }\end{array}$ & 8 & 4 & 3 & 3 & 2 & 4 & 3 & 6 & \\
$\begin{array}{l}\text { TBR culprit } \\
\text { TBR non- }\end{array}$ & 2.45 & 2.72 & 2.27 & 2.40 & 1.33 & 1.88 & 2.14 & 1.70 & $\begin{array}{l}2.11^{* *} \\
(0.42)\end{array}$ \\
$\begin{array}{l}\text { TBRrit } \\
\text { culprit }\end{array}$ & 1.57 & 1.61 & 1.14 & 1.08 & 0.80 & 1.37 & 1.73 & 1.62 & $\begin{array}{l}1.36^{* *} \\
(0.30)\end{array}$ \\
\hline
\end{tabular}

TBR=Tissue-to-Background Ratio, $\mathrm{RPDA}=$ right posterior descending artery, $\mathrm{LAD}=$ left anterior descending artery, $\mathrm{RCA}=$ right coronary artery, $\mathrm{ROI}=$ region of interest, $* *$ p-value $<0.01$

Table 3. Per patient comparison of the scar and myocardium Tissue-to-Background ratio

\begin{tabular}{lllllllllllll}
\hline Patients & $\mathbf{1}$ & $\mathbf{2}$ & $\mathbf{3}$ & $\mathbf{4}$ & $\mathbf{5}$ & $\mathbf{6}$ & $\mathbf{7}$ & $\mathbf{8}$ & $\mathbf{9}$ & $\mathbf{1 0}$ & $\begin{array}{l}\text { Mean } \\
\text { (SD) }\end{array}$ \\
\hline $\begin{array}{lllllllll}\text { Scar \% } \\
\text { PCI to Scan }\end{array}$ & 4.7 & $\mathrm{NA}$ & 28.1 & 20.6 & 16.6 & 13.1 & 13.9 & 41.4 & 16.6 & 11.2 & $\begin{array}{l}18.4 \\
(10)\end{array}$ \\
(days) & 20 & 9 & 11 & 20 & 15 & 24 & 12 & 12 & 16 & $\begin{array}{l}15.1 \\
(4.6)\end{array}$ \\
\hline $\begin{array}{l}\text { SUV Scar } \\
\text { PET/CT }\end{array}$ & - & NA & 0.68 & 0.61 & 0.36 & - & 0.48 & 0.40 & 0.48 & 0.48 & $\begin{array}{l}0.50 \\
(0.10)\end{array}$ \\
$\begin{array}{l}\text { SUV Myo. } \\
\text { PET/CT }\end{array}$ & - & 0.37 & 0.49 & 0.48 & 0.28 & - & 0.45 & 0.35 & 0.40 & 0.41 & $\begin{array}{l}0.40 \\
(0.06)\end{array}$ \\
$\begin{array}{l}\text { TBR Scar } \\
\text { PET/CT }\end{array}$ & - & NA & 1.01 & 1.00 & 0.89 & - & 0.75 & 0.81 & 0.75 & 0.90 & $\begin{array}{l}0.87 * * \\
(0.10)\end{array}$ \\
$\begin{array}{l}\text { TBR Myo. } \\
\text { PET/CT }\end{array}$ & - & 0.72 & 0.73 & 0.79 & 0.70 & - & 0.71 & 0.71 & 0.62 & 0.78 & $\begin{array}{l}0.72^{* *} \\
(0.05)\end{array}$ \\
\hline $\begin{array}{l}\text { SUV Scar } \\
\text { PET/MR }\end{array}$ & 1.41 & NA & 0.90 & 0.76 & 0.61 & 1.38 & 0.64 & 0.77 & 0.76 & 0.70 & $\begin{array}{l}0.88 \\
(0.28)\end{array}$ \\
$\begin{array}{l}\text { SUV Myo. } \\
\text { PET/MR }\end{array}$ & 1.09 & 0.57 & 0.82 & 0.70 & 0.53 & 1.15 & 0.62 & 0.69 & 0.69 & 0.62 & $\begin{array}{l}0.75 \\
(0.20)\end{array}$ \\
$\begin{array}{l}\text { TBR Scar } \\
\text { PET/MR }\end{array}$ & 0.98 & NA & 0.83 & 0.83 & 0.90 & 0.86 & 0.66 & 0.82 & 0.76 & 0.65 & $\begin{array}{l}0.81^{*} \\
(0.10)\end{array}$ \\
$\begin{array}{l}\text { TBR Myo } \\
\text { PET/MR }\end{array}$ & 0.76 & 0.73 & 0.76 & 0.76 & 0.78 & 0.72 & 0.64 & 0.73 & 0.69 & 0.58 & $\begin{array}{l}0.72^{*} \\
(0.06)\end{array}$ \\
\hline
\end{tabular}

TBR=Tissue-to-Background Ratio, $* *$ p-value $<0.01, *$ p-value $<0.05$ 

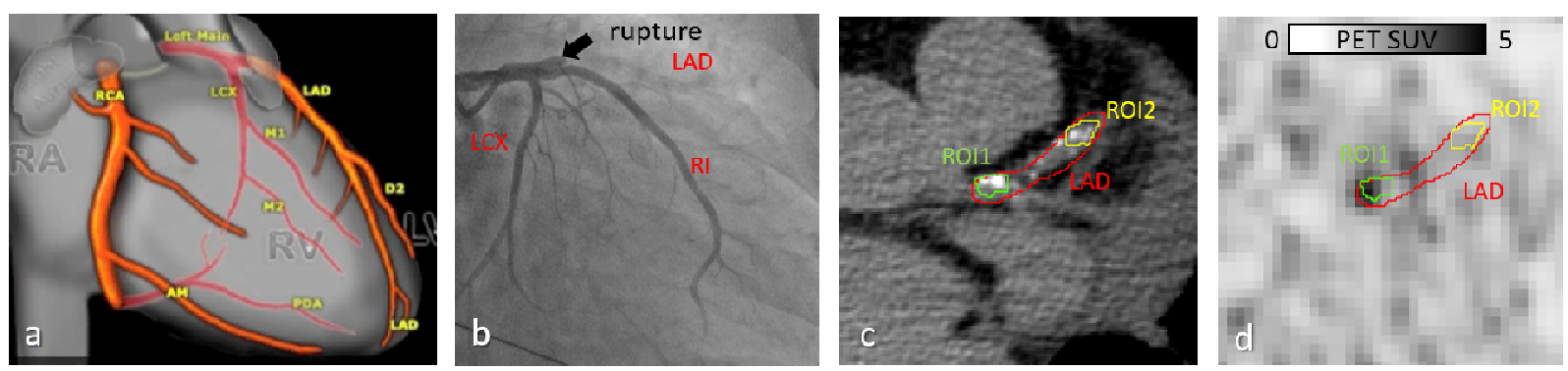

Fig. 1 Example of 18F-NaF PET-CT detection of a ruptured plaque. a) Definition of the coronary arteries, by "Robin Smithuis and Tineke Wilems, the Radiology Assistant". b) Angiogram examination showing plaque rupture and acute occlusion at proximal LAD. (RI represents a large Ramus Intermedius) c) CT calcium scoring images in the axial view where the LAD and 2 ROIs have been segmented. d) Corresponding PET image with the reported segmentations, images were scaled with SUV in range [0-5]. ROI1 corresponds to the ruptured plaque 
1. Dixon SAX resampling

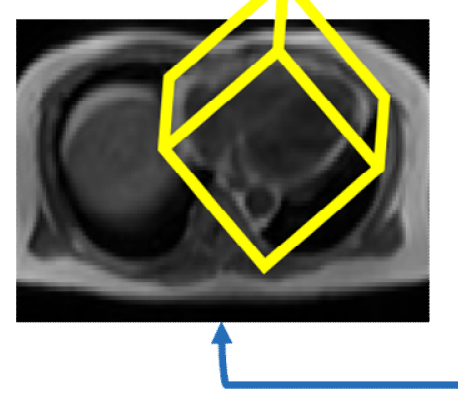

2. Automatic $3 \mathrm{D}$ rigid registration using mutual information

3. Report of ROIs on Dixon and PET/MR images
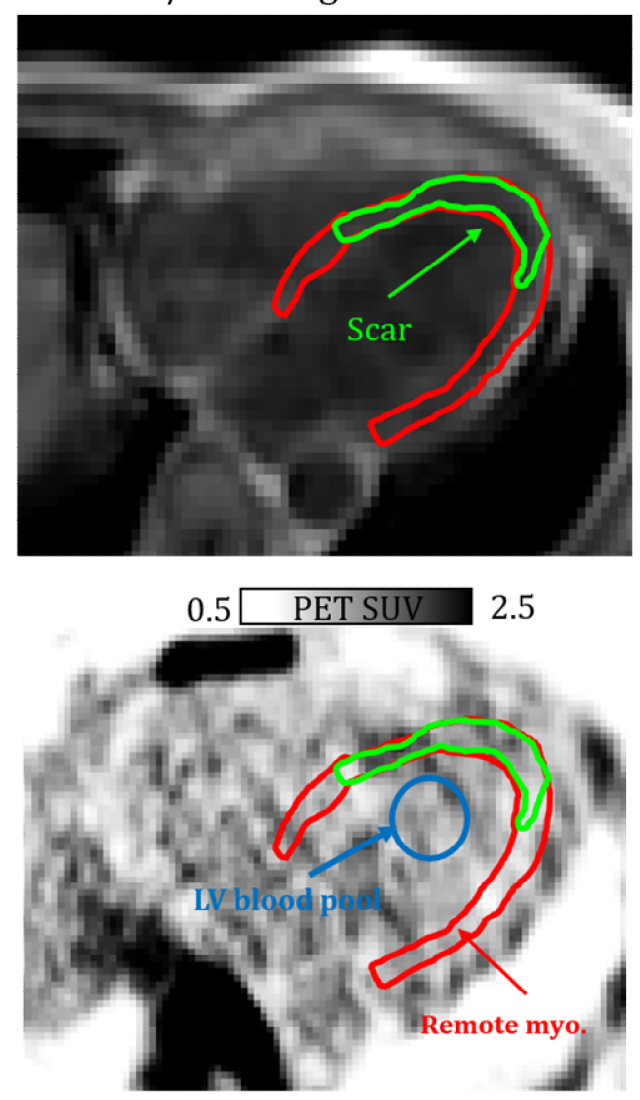

\section{LGE to PET/MR registration}

1. CT SAX resampling

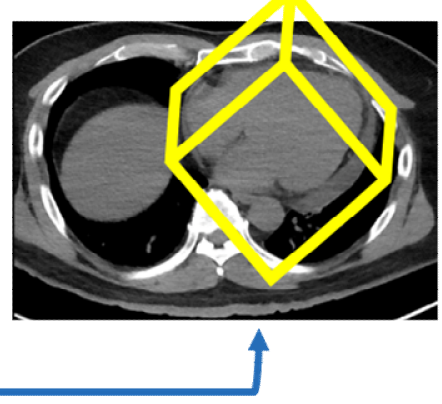

2. Landmark-based rigid registration

3. Report of ROIs on CT and PET/CT images

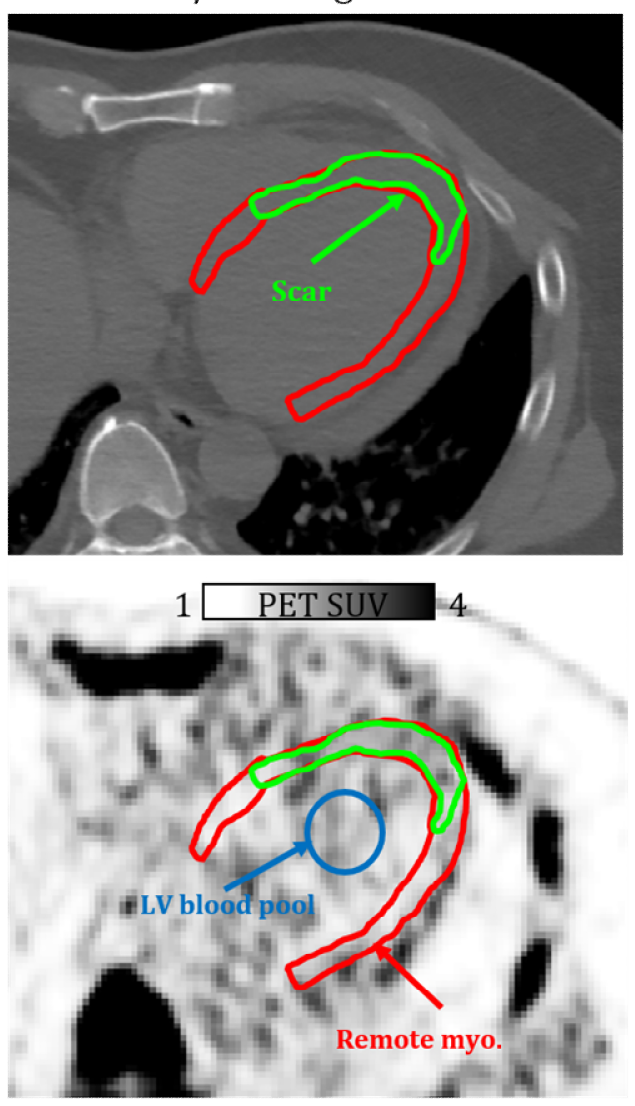

\section{LGE to PET/CT registration}

Fig. 2 (Left: PET/MR) Description of the LGE to PET/MR registration that leads to the measurement of scar Vs heathy myocardium TBR. (Right: PET/CT) For the same patient, description of the LGE to PET/CT registration. With both modalities, higher uptake is noticeable in the scar (green area) compared to the remote myocardium (red) 

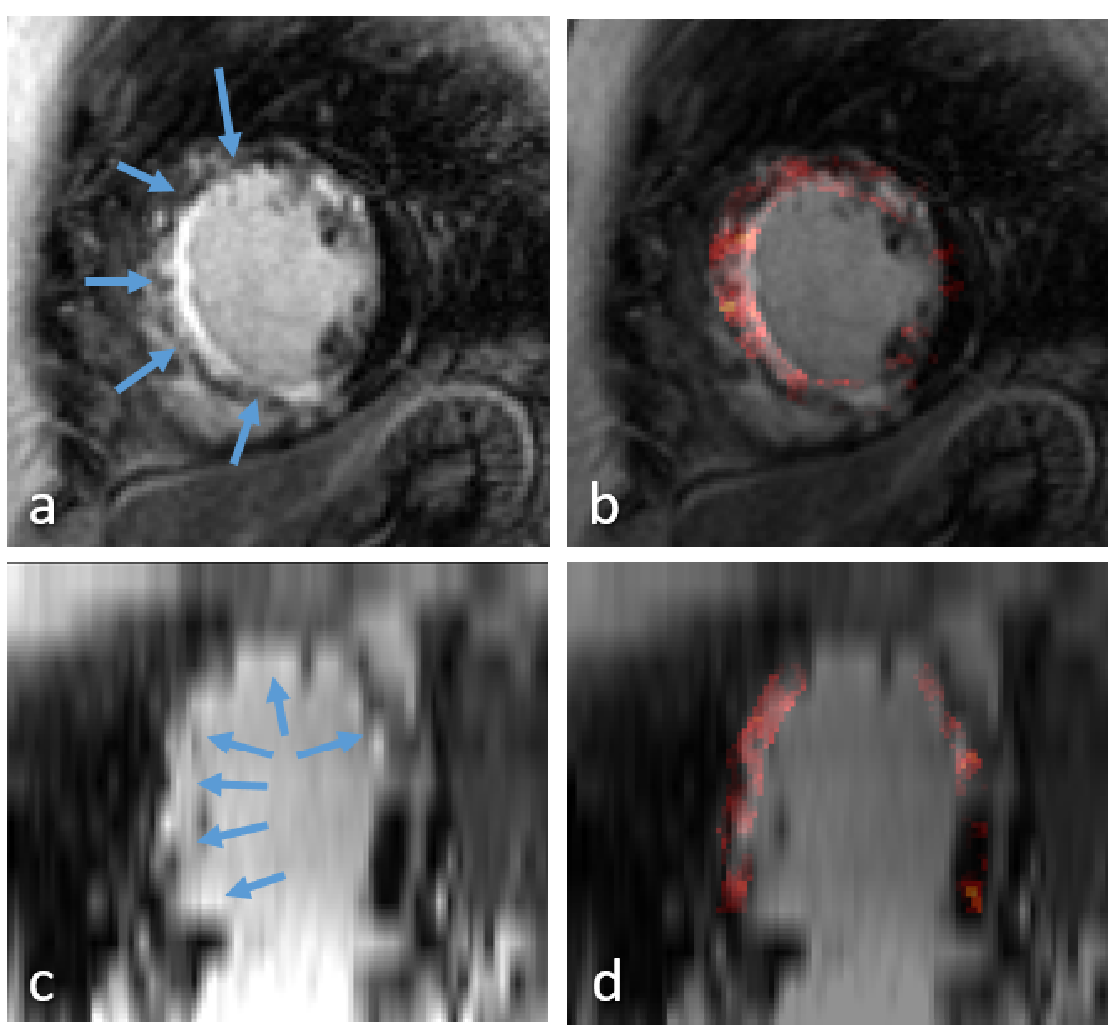

Fig. 3 Example of LGE image showing extensive infarct tissue (arrows) in short-axis (a) and 2chamber (c) views. ${ }^{18} \mathrm{~F}-\mathrm{NaF}$ PET uptake was overlaid on the myocardium muscle on images (b) and (d) respectively showing increased uptake in the infarct tissue compared to healthy myocardium
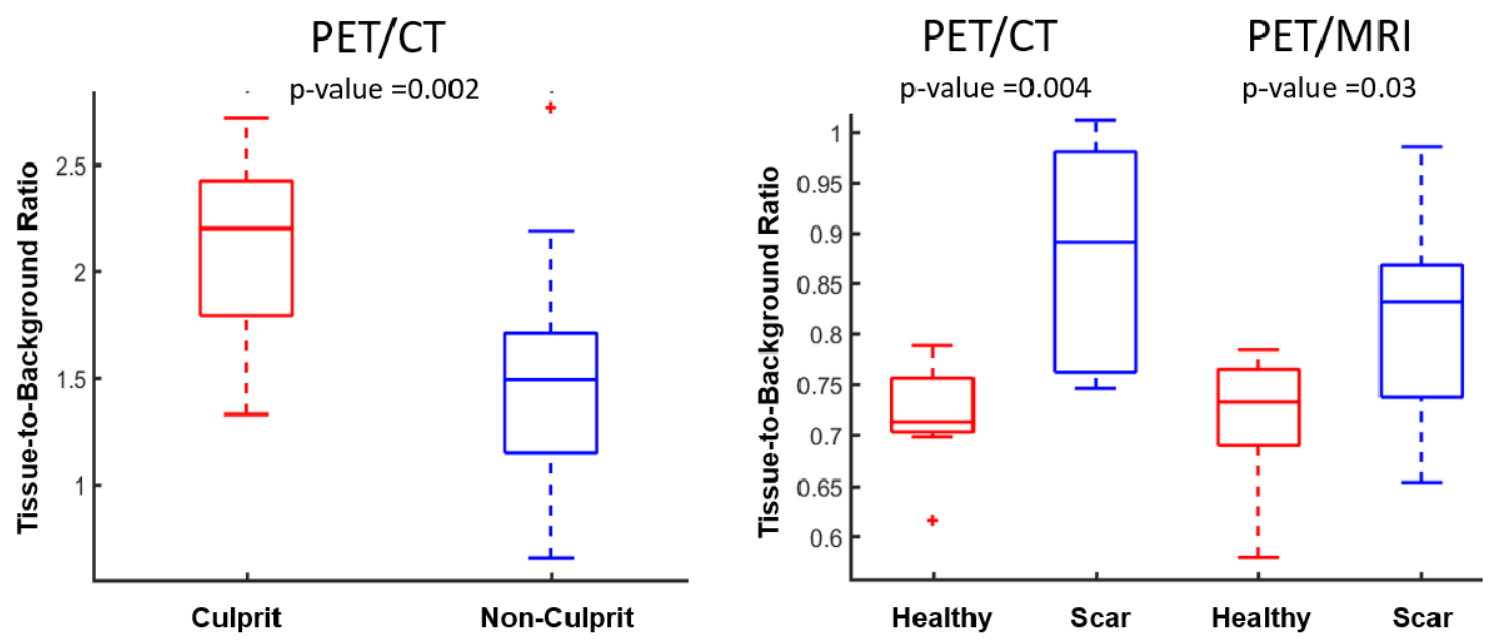

Fig. 4 (Left) Boxplot representation of the 2 groups of lesions showing a significant difference in TBR between the culprit and the non-culprit lesions. (Right) Boxplot representation of the myocardium uptake using both the PET/CT and the PET/MR modalities 